\title{
Volumetric Properties of Secondary Butanol and Tertiary Butanol in Water and Aqueous Micellar Systems of Sodium Dodecyl Sulphate
}

\author{
J. Alauddin ${ }^{1}$, S. Pande ${ }^{2}$, S. C. Mohanta ${ }^{2}$, M. Alauddin ${ }^{1,2, *}$ \\ ${ }^{1}$ Department of Applied Chemistry and Chemical Engineering, Islamic University, Kushtia- \\ 7003, Bangladesh \\ ${ }^{2}$ Department of Chemistry, Jashore University of Science and Technology, Jashore-7408, \\ Bangladesh
}

Received 24 December 2019, accepted in final revised form 17 April 2020

\begin{abstract}
The apparent molal volumes of $s e c$-butanol and $t$-butanol in water and in aqueous micellar system of sodium dodecyl sulphate have been determined from density measurements at different surfactant concentrations and temperatures. The partial molal volumes of the alcohols in aqueous micellar system at infinite dilution, $\overline{V_{2}^{0}}$ (mic) were obtained from apparent molal volume data and compared with the corresponding values in aqueous solvent, $\overline{V_{2}^{0}}(\mathrm{aq})$. The standard partial molal expansibilities, $\overline{E_{2}^{0}}$ of the alcohols were evaluated from $\overline{V_{2}^{0}}$ data at various temperatures. The transfer apparent molal volumes, $\Delta \phi_{t r}^{0}$ for the alcohols from water to surfactant-water system are determined from apparent molal volume data. The sign and magnitude of these parameters are used to analyze the location of the solubilizate (alcohols) in the micellar system and the nature of interactions between alcohols and the micellar aggregates.
\end{abstract}

Keywords: Surfactant; Micelles; Solubilization; Partial molal volume.

(C) 2020 JSR Publications. ISSN: 2070-0237 (Print); 2070-0245 (Online). All rights reserved. doi: http://dx.doi.org/10.3329/jsr.v12i3.44614

J. Sci. Res. 12 (3), 419-429 (2020)

\section{Introduction}

The properties of solution can be accounted in terms of solvent-solvent, solvent-solute and solute-solute interactions. In terms of thermodynamics, the concentration dependence of a given property is a measure of solute-solute interactions, whereas the same property extrapolated to the limit of infinite dilution provides a measure of solute-solvent interactions. The partial molar volume of a solute at infinite dilution reflects the intrinsic volume of the solute and the volume change resulting from solutesolvent interactions. Thus any change in this property of a solute with composition of mixed solvent and temperature reflects the change of interactions of solute particles

* CorrespondenceAuthor: $\underline{\text { malauddin.kst@gmail.com }}$ 
and their local environment. The partial molar volume of any component in a solution can be readily obtained from concentration dependence of its apparent molar volume. Solute-water interactions or hydration phenomena can be conveniently classified as hydrophilic hydration where solute-solvent interaction occurs via $\mathrm{H}$-bonding, ionic hydration where interactions occur through electrostatic forces and hydrophobic hydration which is observed in solutions essentially for apolar molecules such as alcohols, esters, amines etc. Inspite of a tremendous amount of works done on the properties of water and aqueous solutions, the solutions of non-electrolytes and organic solutes are of special interest since many of these solutes are known to promote the structure of water [1]. It is accepted that in the vicinity of nonpolar molecules or nonpolar groups there are strengthening of hydrogen bonds between water molecules. This hydration is quite different from the usual solute-solvent interaction and since it is essentially a measure of the increase in solvent-solvent interaction brought about by hydrophobic part of the molecule, it is termed as hydrophobic hydration. In many aqueous solutions, the non-polar part of the molecules or ions tends to associate by partially or completely removing from the contact with the solvent. This process of interaction of two or more hydrated polar molecules, known as hydrophobic interactions is believed to contribute to the maintenance of many ordered macromolecular structures. The studies of solution properties of such molecules in aqueous media are, therefore, of fundamental interest.

Surfactants are known to play a vital role in many processes of interest in both fundamental and applied science. The most outstanding property of surfactant solution is their ability to solubilize organic compounds which are insoluble or sparingly soluble in water [2]. The increased solubility at concentration above the critical micelle concentration $(\mathrm{cmc})$, known as solubilization is due to formation of micelles [3] by surfactant monomers. These provide a more compatible environment for solubilization of hydrophobic molecules. It is primarily for this reason that surfactant find useful applications in many biological, pharmaceutical and industrial processes. The primary interest concern the extent to which a particular compound can be solubilized in a given surfactant solution at a specified concentration. The other aspect of major interest is to know the regions where the hydrophobic molecules locate within the micelles.

The surfactant-water-alcohol ternary systems have attracted much interest in recent years [4-7] since these systems in the presence of oil can led to micro-emulsions that have important applications in enhanced oil recovery. As well, since lipid bilayers are somewhat similar to micelles in terms of their physicochemical properties, micellar solubilization can be used as a model system to provide useful insight into the understanding of such processes in these biological systems. In view of its importance, the solubilizations of various hydrophobic molecules in different surfactant solutions have been investigated over the years [8-10]. The major interest is to know the regions where the solubilizate molecules locate in the micellar aggregates and the nature of interactions of the additives with micellar aggregates. In the present work the apparent 
molal volume, $\phi_{v}$ of sec-butanol and $t$-butanol in water and in aqueous micellar system of surfactant were measured from density measurement. The partial molal volume of these additives in water and surfactant solution at infinite dilution, $\overline{V_{2}^{0}}$ were obtained from apparent molal volume data. The partial molal volume of alcohols dissolved in aqueous micellar system of sodium dodecyl sulphate (SDS) is compared with corresponding values in pure water to predict the environment of alcohols in the micellar aggregates and the nature of interactions between solubilizates and micelles.

It is well known that alcohols and hydrocarbons form mixed micelles with surfactant in water [11-13]. Thermodynamic properties such as cmc, the size, shape and degree of dissociation of micelles are all changed in the presence of these hydrophobic compounds. These systems can be studied through thermodynamic function of transfer of one of the solute from water to an aqueous solution of another solute [14-17]. Generally, the standard thermodynamic function for the transfer of as reference solute 3 from water (component 1) to aqueous solution of surfactant $(1+2)$ is given by,

$$
\overline{\Delta Y_{3}^{0}}(1 \rightarrow 1+2)=\overline{\Delta Y_{3}^{0}}(1+2)-\overline{\Delta Y_{3}^{0}}(1)
$$

In this case $\overline{\Delta Y_{3}^{0}}(1)$ is the standard partial molal quantity of solute in water and $\overline{\Delta Y_{3}^{0}}(1+2)$ is the same function in aqueous surfactant solution. A number of attempts [15-17] have been made to treat quantitatively the thermodynamics of ternary micellar systems, mostly in relation to the solubilization phenomenon. When mixed micelles are formed upon the solubilization of an additive in the micellar aggregates, the thermodynamic transfer properties such as volume, enthalpy, compressibility, heat capacity change significantly.

The transfer apparent molar volume $\Delta V_{t r}$ of solubilizates from water to surfactantwater mixed solvent can be calculated from

$$
\Delta V_{t r}=\phi_{v}(w+s)-\phi_{v}(w)
$$

where $\phi_{v}(\mathrm{w}+\mathrm{s})$ is the apparent molal volume of alcohol in SDS-water mixture and $\phi_{v}(\mathrm{w})$ is the apparent molal volume of alcohol in water. In the present work, the transfer volume of $s e c$-butanol and $t$-butanol from water to SDS micelle of different concentrations were estimated in order to predict the solubilization process of this additive.

\section{Experimental}

SDS was obtained from Merch-Schuchardt, Germany. Its purity was $>99 \%$ and used without further purification. The cmc value of SDS was found to be $0.008 \mathrm{molkg}^{-1}$ at $25{ }^{\circ} \mathrm{C}$ which is close to the values reported in the literature [18]. Secondary butanol $(\sec -\mathrm{BuOH})$, and tertiary butanol $(t-\mathrm{BuOH})$ were obtained from E. Merck, Germany. 
Their purity was $>99.5 \%$. All these compounds were of reagent grade and used without further purification. Water used in this experiment was double distilled. All the solutions were prepared by weight and their concentrations are expressed as number of solute moles per kg of solvent. Aqueous stock solutions of SDS of 0.15, 0.25 and $0.35 \mathrm{~mol} \mathrm{~kg}^{-1}$ were prepared and used as mixed solvent in the measurement of apparent molar volume. In the measurement of $\phi_{v}$ of alcohols in mixed solvent, the solutions were stirred for several hours to ensure that equilibrium conditions reached and the temperature was controlled by a thermostatic water bath. The system was capable of monitoring the temperature with a precision of $\pm 0.005{ }^{\circ} \mathrm{C}$.

The apparent molar volume of solutions was calculated from the relation

$$
\phi_{v}=\frac{1000}{m d d_{0}}\left(d_{0}-d\right)+\frac{M}{d}
$$

where $m$ is the molality ( $m o l \mathrm{~kg}^{-1}$ ) of the solution, $M$ is the molar mass of the solute, $d$ is the density of solution, and $d_{0}$ is the density of the solvent. The density data were obtained by using pyknometer having $25 \mathrm{~mL}$ capacity. The precision in the density data obtained from replicate measurements was found to be better than $\pm 5 \times 10^{-5} \mathrm{~g} \mathrm{~cm}^{-3}$ for our system under experimental conditions.

\section{Results and Discussion}

\subsection{SDS-sec-BuOH system}

The concentration dependence of apparent molal volume, $\phi_{v}$ of sec-BuOH in water and in aqueous micellar system of SDS of $0.15,0.25$ and $0.35 \mathrm{~m}$ at $30,35,40$ and 45 ${ }^{\circ} \mathrm{C}$ are shown in Fig. 1. It appears that the apparent molal volumes of $2-\mathrm{BuOH}$ are very much dependent upon the concentration of both the alcohol and surfactant concentration. The apparent molal volumes of the alcohol both in water and in three different aqueous micellar system of SDS at different temperatures decreases almost linearly with increasing sec-BuOH (Fig. 1). The decrease in $\phi_{v}$ in $s e c-\mathrm{BuOH}$ in water with increasing its concentration indicates that $s e c-\mathrm{BuOH}$ undergoes hydrophobic hydration in aqueous phase. A slight deviation from linearity observed with high alcohol content indicates that sec-BuOH undergoes hydrophobic hydration and alcohol-alcohol interactions become significant with increased alcohol content. The values of $\phi_{v}$ of $s e c-\mathrm{BuOH}$ in aqueous micelle solution of SDS is, however, remarkably higher than its value in water and increases with increasing surfactant concentration. This increase in volume is probably the results of hydrophobic dehydration of alcohol and an increasing proportion of sec-BuOH is transferred into SDS micelle. It is expected that the surfactant forms mixed micelle with sec-BuOH and upon increasing the alcohol concentration the surfactant-alcohol interactions become predominant over alcohol-water interactions and becomes more significant with increasing surfactant concentration. 

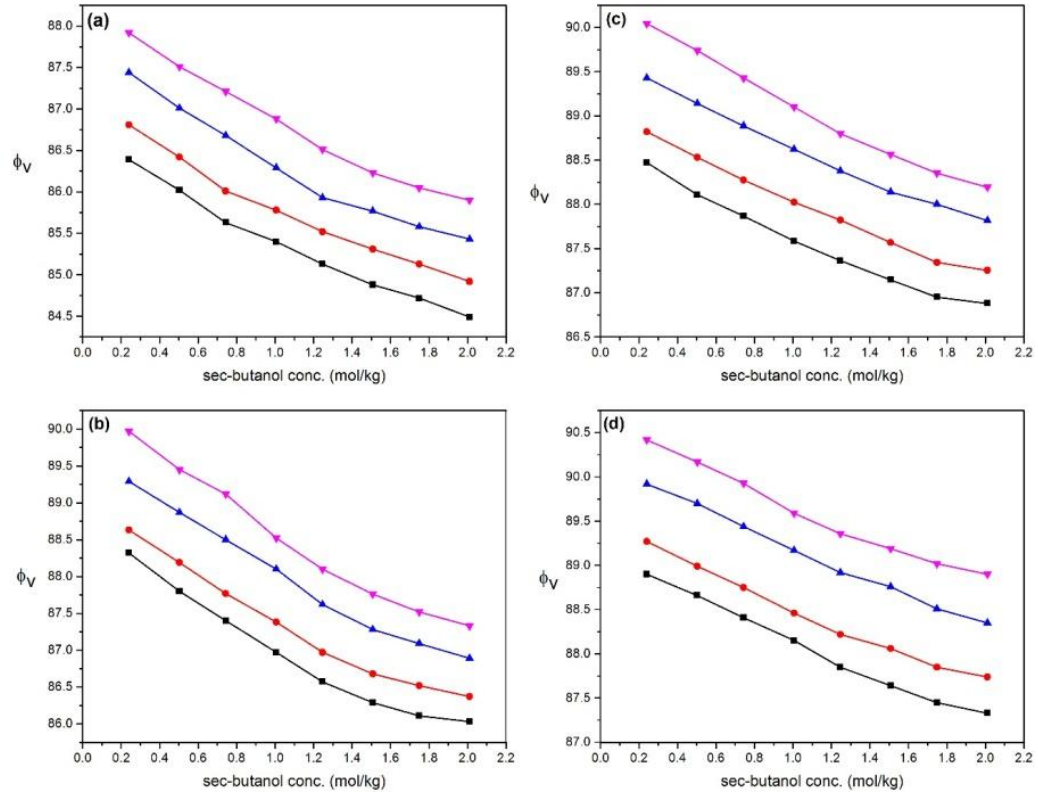

Fig. 1. Apparent molal volume of sec-BuOH in (a) water, (b) $0.15 \mathrm{~m}$ SDS, (c) $0.25 \mathrm{~m}$ SDS and (d) $0.35 \mathrm{~m}$ SDS at different temperatures, $\boldsymbol{\square}, 30 ; \bullet, 35 ; \boldsymbol{\Lambda}, 40$; and $\boldsymbol{\nabla}, 45^{\circ} \mathrm{C}$.

\subsection{SDS-t-BuOH system}

The variation of apparent molal volume, $\phi_{v}$ of $t-\mathrm{BuOH}$ in water and in aqueous micellar system of SDS of different concentrations with the molality of $t-\mathrm{BuOH}$ at 30 , 35,40 and $45{ }^{\circ} \mathrm{C}$ are shown in Fig. 2. The volumetric behavior of $t$-BuOH both in water and surfactant-water solvent systems is found to be different from that observed for $s e c-\mathrm{BuOH}$. In contrast to $s e c-\mathrm{BuOH}$, the apparent molar volume of $t-\mathrm{BuOH}$ increases in water with increasing $t$ - $\mathrm{BuOH}$ molality. Alcohols in water generally undergo hydrophobic hydration with overall economy of space. Hydrophobic hydration promotes the structure of liquid water around the hydrocarbon moieties and they lead to the formation of interstitial cavities surrounding the hydrophobic part of the solutes and the solute fills the interstitial cavities of the structure. This loss of free space during hydrophobic hydration is larger than the increase in volume accompanying the increase of ice-likeness. Alcohols in water are thus accompanied by significant volume contraction. In the case of $t-\mathrm{BuOH}$ it is expected that due to an unfavorable geometric fitting of molecules only a fewer sites are available for the accommodation of solute within interstitial cavities. Moreover, in aqueous system, association of alcohol molecules occurs by hydrophobic interaction with increasing alcohol concentration with subsequent release of some structured hydration water. The overall result is the expansion of volume. 

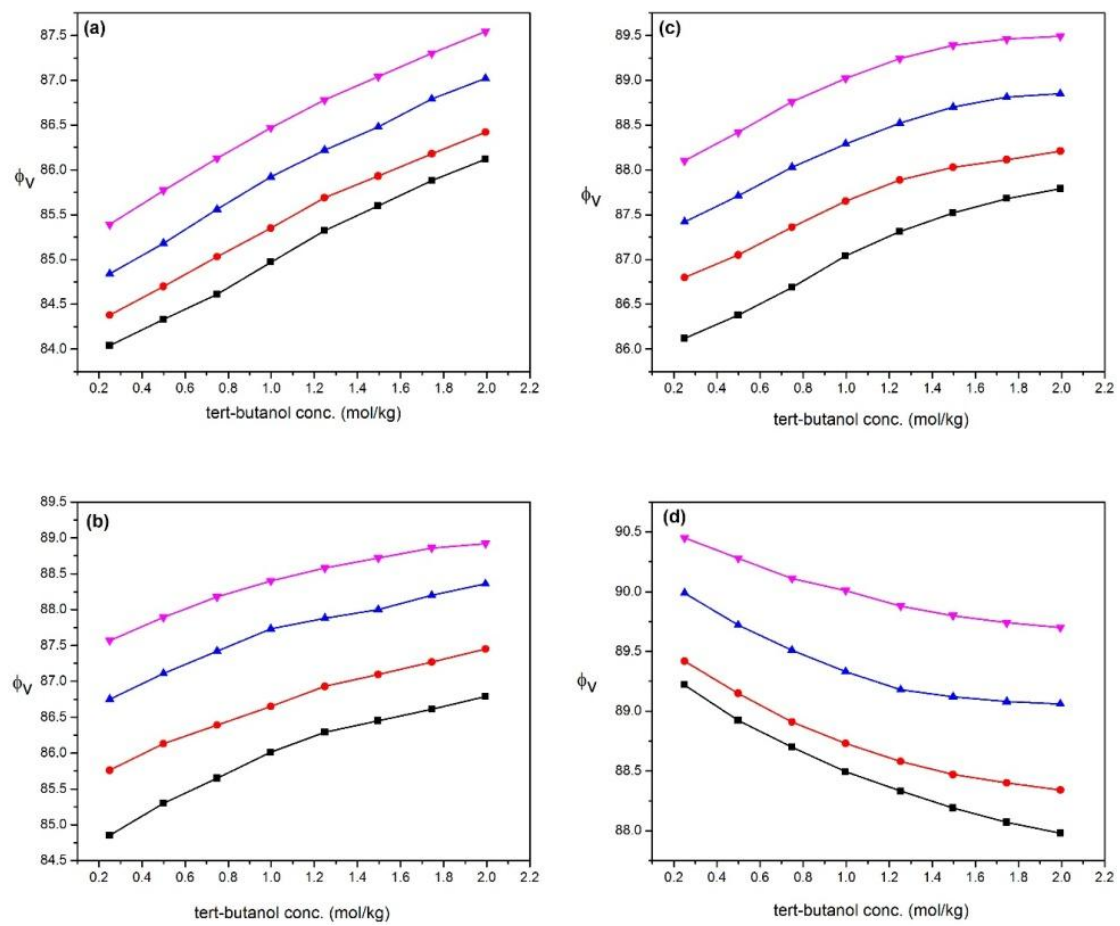

Fig. 2. Apparent molal volume of $t$ - $\mathrm{BuOH}$ at temperature in (a) water, (b) $0.15 \mathrm{~m} \mathrm{SDS}$, (c) 0.25 $\mathrm{m}$ SDS and (d) $0.35 \mathrm{~m}$ SDS at different temperatures, $\mathbf{\bullet}, 30 ; \bullet, 35 ; \boldsymbol{\Lambda}, 40$; and $\boldsymbol{\nabla}, 45^{\circ} \mathrm{C}$.

The apparent molar volume, $\phi_{v}$ of $t$-BuOH dissolved in $0.1 \mathrm{~m}$ and $0.25 \mathrm{~m}$ SDS solutions increase with increasing alcohol concentration and a slight deviation from linearity is observed with higher alcohol concentration at all temperatures. The interior of the micelle is generally considered to be the locus of solubilization for very nonpolar solubilizates such as $n$-alkanes [19]. Solubilizate molecules of relatively high polarity such as alcohols are believed to be solubilized in the interfacial region of the micelle [2]. The volume of hydrophobic solute in a hydrocarbon solvent is generally much higher than its value in water. This is expected for a solute in hydrocarbon solvent as because there is relatively more free space in the vicinity of hydrophobic solute dissolved in a hydrocarbon solvent than water. The results of our studies show that the values of $\phi_{v}$ of $t$ - $\mathrm{BuOH}$ in aqueous micelle solution of SDS are significantly higher than its value in water and increases with increasing surfactant concentration. This suggest that $t$ - $\mathrm{BuOH}$ in SDS solution experience some hydrocarbon-like environment and the alcohol molecules are favorably dispersed in the micellar aggregates forming mixed micelle with increasing surfactant concentration. The slight deviation from linearity indicates that the alcohol molecules initially at low concentration are solubilized close to the surface in the palisade layer and the 
solubilization of excess alcohols occurs by adsorption at the micelle-water interface. The $\phi_{v}$ of $t-\mathrm{BuOH}$ in $0.35 \mathrm{~m}$ SDS solution is much higher than its value in water. This suggests that alcohol molecules in $0.35 \mathrm{~m}$ surfactant solution experience more hydrocarbon-like environment in the system i.e. an increasing proportion of alcohol is being transferred within the micellar aggregates deeper into the palisade layer. The incorporation of the solubilizate into the micelle may change the size and shape of the micelle considerably. Thus the decrease of $\phi_{v}$ with increasing $t$ - $\mathrm{BuOH}$ concentration indicates that the alcohol may induce the growth of SDS micelle. As a result, the total surface area of the micelle is increased and excess alcohol molecules from increased concentration of $t-\mathrm{BuOH}$ are preferentially solubilized close to the micellar surface having mixed solvent environment.

\subsection{Standard partial molal volume, $\overline{V_{2}^{0}}$ and expansibilities, $\overline{E_{2}^{0}}$ of alcohols}

The partial molal volume of sec- $\mathrm{BuOH}$ and $t-\mathrm{BuOH}$ in water and different SDS-water solvent systems at infinite dilution, $\overline{V_{2}^{0}},\left(\phi_{v}^{0}=\overline{V_{2}^{0}}\right)$ were estimated by extrapolation of $\phi_{v}$ values to $\mathrm{m}=0$. Derived values of $\overline{V_{2}^{0}}$ for the alcohol in water and in aqueous micellar system of SDS are shown in Table 1 . The values of $\overline{V_{2}^{0}}$ of the alcohol obtained in this work show a considerable variation with surfactant concentration and temperature.

Table 1. Partial molal volumes, $\overline{V_{2}^{0}}$ of solubilizates in water and different SDS-water mixed solvent system at $30,35,40$ and $45^{\circ} \mathrm{C}$.

\begin{tabular}{llllll}
\hline Solubilizate & Solvent system & $\overline{V_{2}^{0}} \mathrm{~cm}^{3} \mathrm{~mol}^{-1}$ & & \\
\hline \multirow{3}{*}{ sec-BuOH } & water & $30{ }^{\circ} \mathrm{C}$ & $35{ }^{\circ} \mathrm{C}$ & $40{ }^{\circ} \mathrm{C}$ & $4{ }^{\circ} \mathrm{C}$ \\
& $0.15 \mathrm{~m}$ SDS & 86.83 & 87.31 & 87.86 & 88.21 \\
& $0.25 \mathrm{~m}$ SDS & 88.84 & 89.02 & 89.57 & 90.34 \\
& $0.35 \mathrm{~m}$ SDS & 89.06 & 89.22 & 89.73 & 90.44 \\
$t$-BuOH & water & 83.67 & 83.52 & 90.15 & 90.65 \\
& $0.15 \mathrm{~m}$ SDS & 84.38 & 85.44 & 84.43 & 84.97 \\
& $0.25 \mathrm{~m}$ SDS & 85.68 & 86.40 & 87.43 & 87.18 \\
& $0.35 \mathrm{~m}$ SDS & 89.06 & 89.52 & 90.15 & 87.69 \\
\hline
\end{tabular}

The standard partial molal expansibility, $\overline{E_{2}^{0}}$ (mic) of the alcohol in water and in aqueous micellar system of SDS were obtained from the temperature dependence of $\overline{V_{2}^{0}}\left(\overline{E_{2}^{0}}=\mathrm{d} \overline{V_{2}^{0}} / d T\right)$. The results are shown in Table 2. An apparent linear dependence $\overline{V_{2}^{0}}$ (mic) with temperature is observed for alcohols in water and in all surfactant solutions. The plot of partial molal volume, $\overline{V_{2}^{0}}$ of $s e c-\mathrm{BuOH}$ and $t-\mathrm{BuOH}$ in water and in different surfactant-water solvent systems as a function of temperature is shown in Fig. 3. 
Table 2. Standard partial molal expansibilities, $\overline{E_{2}^{0}}$ of sec-BuOH and $t$-BuOH in water and different SDS-water solvent systems.

\begin{tabular}{lll}
\hline Solubilizate & Solvent systems & $\overline{E_{2}^{0}} \mathrm{~cm}^{3} \mathrm{~mol}^{-1} \mathrm{~kg}^{-1}$ \\
\hline sec-BuOH & water & 0.0938 \\
& $0.15 \mathrm{~m}$ SDS & 0.1064 \\
& $0.25 \mathrm{~m}$ SDS & 0.1067 \\
& $0.35 \mathrm{~m}$ SDS & 0.1080 \\
\hline - $\mathrm{BuOH}$ & water & 0.0868 \\
& $0.15 \mathrm{~m}$ SDS & 0.1878 \\
& $0.25 \mathrm{~m}$ SDS & 0.1326 \\
& $0.35 \mathrm{~m}$ SDS & 0.0800 \\
\hline
\end{tabular}
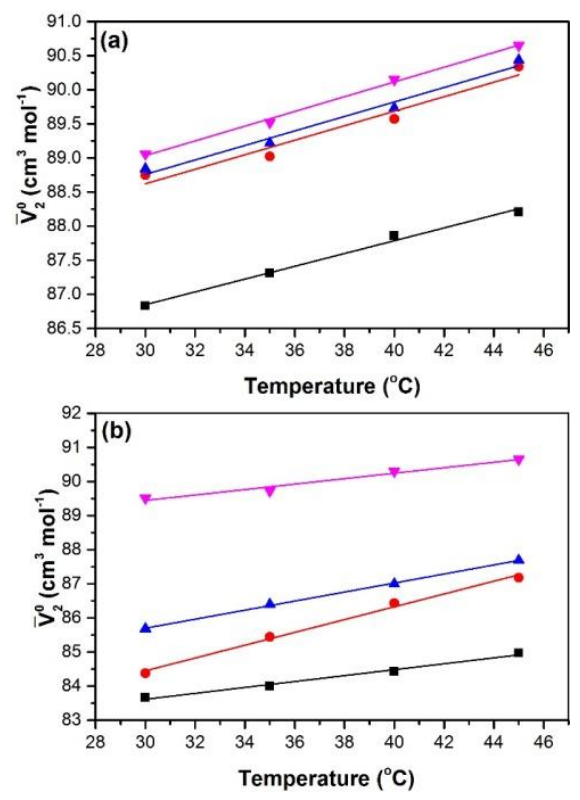

Fig. 3. Standard partial molal volume, $\overline{V_{2}^{0}}$ of (a) $s e c-\mathrm{BuOH}$ (b) $t-\mathrm{BuOH}$ in water and in aqueous micellar system of SDS as a function of temperature, $\boldsymbol{\bullet}$, water; $\bullet, 0.15 \mathrm{~m}$ SDS; $\boldsymbol{\Lambda}, 0.25 \mathrm{~m} \mathrm{SDS}$; and $\boldsymbol{\nabla}, 0.35 \mathrm{~m}$ SDS

The standard partial molal volume, $\overline{V_{2}^{0}}$ reflects the intrinsic volume of the solute and the volume changes resulting from solute-solvent interactions. Thus any change of $\overline{V_{2}^{0}}$ of a solute with composition of a mixed solvent and temperature reflects the change of interactions between the solute particles and its local environment. In the present work, the standard partial molal volume of sec-BuOH and $t-\mathrm{BuOH}$ in aqueous micellar system of SDS, $\overline{V_{2}^{0}}(\mathrm{mic})$ is compared with corresponding values in water, $\overline{V_{2}^{0}}$ to predict the local environment of the solubilizate in micellar aggregates and the nature of interactions between the additive and the micelles. In the case of sec-BuOH, it is observed that $\overline{V_{2}^{0}}$ (mic) is significantly larger than $\overline{V_{2}^{0}}$ (aq) and show a considerable increase with increasing surfactant concentration and temperature. It 
indicates that $\mathrm{sec}-\mathrm{BuOH}$ experiences some hydrocarbon like environment and that it is primarily solubilized close to the micellar surface with short penetration into the micellar aggragates and gets more into the palisade layer upon increasing surfactant concentration and temperature. The values of $\overline{V_{2}^{0}}$ (mic) for $t$ - $\mathrm{BuOH}$ shows that it is considerably larger than its value in water, $\overline{V_{2}^{0}}(\mathrm{aq})$, but this difference between $\overline{V_{2}^{0}}$ (mic) and $\overline{V_{2}^{0}}(\mathrm{aq})$, however, depends on surfactant concentration and temperature. This suggests that this molecule is likely to be solubilized within the palisade layer of the micelle with its polar $-\mathrm{OH}$ group directing towards the surrounding water. It is observed that the values of $\overline{V_{2}^{0}}$ (mic) for $t$ - $\mathrm{BuOH}$ dissolved in $0.35 \mathrm{~m}$ SDS is $4-5 \mathrm{~cm}^{3}$ $\mathrm{mol}^{-1}$ larger than those obtained in water, $0.15 \mathrm{~m}$ and $0.25 \mathrm{~m}$ SDS solution at different temperatures. The larger values of $\overline{V_{2}^{0}}$ (mic) in the case of $0.35 \mathrm{~m}$ SDS further reflects that $t$ - $\mathrm{BuOH}$ experiences more hydrocarbon-like environment in this surfactant system and is preferentially solubilized deeper into the palisade layer of the micelle. It appears that depending upon the concentration and temperature both the alcohols are favorably dispersed in the micellar aggregares and $t \mathrm{BuOH}$ experience more hydrocarbon-like environment than $s e c-\mathrm{BuOH}$.

The temperature dependence of $\overline{V_{2}^{0}}$ gives a direct measure of partial molal expansibility, $\overline{E_{2}^{0}}$ at infinite dilution. The expansibility of hydrophobic solute in water is, generally, positive but small since there is less free space in the cosphere of hydrophobic solute in water. The relatively larger values of $\overline{E_{2}^{0}}$ is thus expected for a solute in a hydrocarbon solvent where there is relatively more free space in the vicinity of hydrophobic solute. The results of our studies show that the values of $\overline{E_{2}^{0}}$ for sec$\mathrm{BuOH}$ and $t-\mathrm{BuOH}$ in water and surfactant solution are positive. The values $\overline{E_{2}^{0}}$ (mic) for both the alcohol is higher than its value in water, $\overline{E_{2}^{0}}(\mathrm{aq})$ with an exception for $t$ $\mathrm{BuOH}$ in $0.35 \mathrm{~m}$ SDS solution. The variation of the magnitude of $\overline{E_{2}^{0}}$ (mic) with increasing surfactant concentration does not occur in a consistent manner for the alcohols. In the case of $2-\mathrm{BuOH}$ the values of $\overline{E_{2}^{0}}$ (mic) increases with increasing surfactant concentration while for $t-\mathrm{BuOH}$ it decreases. Nevertheless, the values of $\overline{E_{2}^{0}}$ (mic) indicate that both the alcohols experience hydrocarbon-like environment in the surfactant solution. It is expected that micelles at high surfactant concentration may not retain its spherical shape. The shape change and expected increase in the surface area of the micelles which makes the micelles more hydrated at $0.35 \mathrm{~m}$ SDS reduce the expansibility.

\subsection{Transfer apparent molal volume, $\Delta \phi_{\text {tr }}^{0}$ of alcohols}

One of the important parameters to investigate the solubilization behavior and interactions in multicomponent system is the transfer volume of solubilizates from water to aqueous micellar system of surfactant. The transfer of apparent molar volume at infinite dilution, $\Delta \phi_{t r}^{0}$ of $s e c-\mathrm{BuOH}$ and $t-\mathrm{BuOH}$ from water to SDS solutions can be calculated from the following equation:

$$
\left.\Delta \phi_{t r}^{0}=\phi_{v}^{0}(\text { water }+ \text { SDS })-\phi_{v}^{0} \text { (water }\right)
$$


where $\phi_{v}^{0}$ (water + SDS) is the apparent molar volume of alcohols in aqueous micelle solution and $\phi_{v}^{0}$ (water)is the apparent molar volume of alcohols in water at infinite dilution. The calculated transfer volume of sec-BuOH and $t-\mathrm{BuOH}$ from water to aqueous micellar system of SDS of different concentrations at $30,35,40$ and $45{ }^{\circ} \mathrm{C}$ is shown in Table 3.

Table 3. Transfer apparent molar volume at infinite dilution, $\Delta \phi_{t r}^{0}$ of $s e c-\mathrm{BuOH}$ and $t-\mathrm{BuOH}$ from water to SDS-Water solvent systems at $30,35,40$ and $45{ }^{\circ} \mathrm{C}$.

\begin{tabular}{|c|c|c|c|c|c|}
\hline \multirow[t]{2}{*}[\mathrm{SDS}]{$\mathrm{mol} \mathrm{kg}^{-1}$} & Solubilizate & \multicolumn{4}{|c|}{$\Delta \phi_{t r}^{0} \mathrm{~cm}^{3} \mathrm{~mol}^{-1}$} \\
\hline & \multirow{3}{*}{$s e c-\mathrm{BuOH}$} & $30^{\circ} \mathrm{C}$ & $35^{\circ} \mathrm{C}$ & $40^{\circ} \mathrm{C}$ & $45^{\circ} \mathrm{C}$ \\
\hline 0.15 & & 1.92 & 1.71 & 1.71 & 2.13 \\
\hline 0.25 & & 2.01 & 1.91 & 1.87 & 2.23 \\
\hline 0.35 & \multirow{4}{*}{$t-\mathrm{BuOH}$} & 2.23 & 2.21 & 2.29 & 2.44 \\
\hline 0.15 & & 0.71 & 1.45 & 2.00 & 2.21 \\
\hline 0.25 & & 2.01 & 2.41 & 2.57 & 2.72 \\
\hline 0.35 & & 5.39 & 5.53 & 5.72 & 5.68 \\
\hline
\end{tabular}

It appears that the values of $\Delta \phi_{t r}^{0}$ for the transfer of both sec-BuOH and $t-\mathrm{BuOH}$ from water to SDS-water solutions are positive and the magnitude of $\Delta \phi_{t r}^{0}$ generally increases with increasing surfactant concentration at different temperatures. This indicates that the alcohol molecules are favorably transferred into the hydrocarbon part of the micellar aggregates and experience more hydrocarbon-like environment in the micellar system. Thus the hydrophobic-hydrophobic interactions between surfactant monomers and alcohol molecules increases while the alcohol-water hydrophilic interactions decreases and it is expected that the interaction of counter ions $\left(\mathrm{Na}^{+}\right)$with water is significantly increased. The relatively larger values of $\Delta \phi_{t r}^{0}$ for $t-\mathrm{BuOH}$ dissolved in $0.35 \mathrm{~m}$ SDS solution indicates that is preferentially solubilized deeper into the palisade layer of the micelles at this higher surfactant concentration probably due its shape transition.

\section{Conclusion}

We find that the apparent molal volumes of sec-butanol and $t$-butanol dissolved in both water and surfactant solutions depend on the alcohol concentration and temperature. The solubilization of increased amount of alcohols in different SDSwater solvent system with increased surfactant concentration indicates that alcohols initially undergoes hydrophobic hydration in aqueous phase and hydrophobic dehydration of alcohols occurs upon increasing surfactant concentration of the solution. As a result an increasing amount of alcohols are transferred into micellar aggregates with increasing surfactants concentration and temperature. It appears that both alcohols are favorably dispersed in the micellar aggregates but $t$-butanol experience more hydrocarbon-like environment than sec-butanol, forming mixed micelles. 


\section{References}

1. G. Nemethy and H. A. Scheraga, J. Chem. Phys. 36, 3401 (1962). https://doi.org/10.1063/1.1732473

2. P. H. Elworthy, A. T. Florence, and C.B. McFarlane, Solubililization by Surfactant Active Agents (Chapman and Hall: London, England, 1968).

3. G. S. Hartley, Aqueous Solutions of Paraffin-Chain Salts (Herman, Paris, 1936).

4. M. Gradzielski, K. Horbaschek, and B. Deme, Ind. Eng. Chem. Res. 58, 2596 (2019). https://doi.org/10.1021/acs.iecr.8b05232

5. K. Śliwa, P. Śliwa, E. Sikora, J. Ogonowski, J. Oszmiański, and P. Nowicka, J. Surfactants Deterg. 22, 655 (2019). https://doi.org/10.1002/jsde.12237

6. Y. Umemoto, S. Uchida,T. Yoshida, K. Shimada, H. Kojima,A. Takagi, and N. Namiki, J. Drug Deliv. Sci. Technol. 55, ID 101401 (2019). https://doi.org/10.1016/j.jddst.2019.101401

7. J. Karayil, J. Y. Talmon, P.A. Hassan, B. V. R. Tata, and L. Sreejith, Colloid Polym. Sci. 296, 595 (2018). https://doi.org/10.1007/s00396-017-4252-0

8. A. Kumar, G. Kaur, S. Kansal, G. R. Chaudhary, and S. Mehta, Food Chem. 199, 660 (2016). https://doi.org/10.1016/j.foodchem.2015.12.077

9. Z. Vinarov, V. Katev, D. Radeva, S. Tcholakova, and N. Denkov, Drug Dev. Ind. Pharm. 44, 677 (2018). https://doi.org/10.1080/03639045.2017.1408642

10. M. Alauddin and R. E. Verrall, J. Phys. Chem. 91, 3724 (1989). https://doi.org/10.1021/j100346a070

11. N. Nazir, M.S. Ahanger, and A. Akbar, J. Disper.Sci. Technol. 30, 51 (2009). https://doi.org/10.1080/01932690802477264

12. S. S. Joshi, and S.S. Bhagwat, J. Surface Sci. Technol. 29, 1 (2013).

13. Y. Moro, Mixed Micelle Formation (Springer, New York, 1992). https://doi.org/10.1007/978-1-4899-0700-4_10

14. G. Perron, R. Delisi, I. Davidson, S. Genereux, and J. E. Desnoyers, J. Colloid Interf. Sci. 79, 432 (1981). https://doi.org/10.1016/0021-9797(81)90094-1

15. G. R. Desgranges, A. H. Roux, J. P. Grolier, and A. Viallard, J. Solution Chem. 11, 357 (1982).

16. V. Majer, A. H. Roux, G. R. Desgranges, and A. Viallard, Can. J. Chem. 61, 139 (1982). https://doi.org/10.1139/v83-025

17. R. DeLisi, V. T. Liveri, M. Castangnolo, and A. Inglese, J. Solution Chem. 15, 23 (1986). https://doi.org/10.1007/BF00644596

18. G. Peron and J. E. Desnoyers, J. Chem. Therm. 13, 1105 (1981). https://doi.org/10.1016/0021-9614(81)90009-4

19. E. Vikingstad and H. Høiland, J. Colloid Interf. Sci. 64, 510 (1978). https://doi.org/10.1016/0021-9797(78)90392-2 